\title{
Influência da salinidade no crescimento de juvenis de Jenynsia multidentata Jenyns (Pisces)
}

\author{
Ana C. G. Mai ${ }^{1}$, Alexandre M. Garcia ${ }^{2} \&$ João P. Vieira ${ }^{1}$ \\ ${ }^{1}$ Laboratório de Ictiologia, Departamento de Oceanografia Biológica, Fundação Universidade Federal do Rio Grande. Caixa \\ Postal 474, 96201-900 Rio Grande, Rio Grande do Sul, Brasil. E-mail: anaceciliamai@hotmail.com \\ 2 Pós Doutorado CNPq, Instituto de Pesquisas Hidráulicas, Universidade Federal do Rio Grande do Sul. Caixa Postal 15029, \\ 91501-970 Porto Alegre, Rio Grande do Sul, Brasil. E-mail: amgarcia@mikrus.com.br
}

ABSTRACT. Salinity influence on the growth of Jenynsia multidentata Jenyns (Pisces). Previous field studies suggest that Jenynsia multidentata Jenyns, 1842 populations reach larger sizes in the estuarine region of the Patos Lagoon when compared to the adjacent freshwater environments. A laboratorial experiment was carried out in order to test possible differences in growth rate, total length and average weight of individuals kept in two different salinities (zero and 16). Right after birth, four offspring of different females were split into separate aquariums of four liters each, being that half of the individuals of each offspring was submitted to salinity zero and the another half to salinity 16 . Four aquariums were kept at the constant temperature of $28^{\circ} \mathrm{C}$, whereas the other ones suffered a change in temperature (from 28 to $24^{\circ} \mathrm{C}$ ) that occur 18 days from the beginning of the experiment. After thirtyeight days, all the individuals were measured and weighed. Individuals kept in constant temperature and salinity zero showed a significantly lower total length $\left(\mathrm{TL}_{16}=23.5 \mathrm{~mm} ; \mathrm{TL}_{0}=20.4 \mathrm{~mm} ; \mathrm{p}<0.05\right)$ and weight $\left(\mathrm{W}_{16}=0.151\right.$ $\left.\mathrm{g} ; \mathrm{W}_{\mathrm{o}}=0.086 \mathrm{~g} ; \mathrm{p}<0,05\right)$, when compared with the ones kept in salinity 16 . Those individuals kept in aquariums that suffered variation in temperature also had significantly differences in both response variables $\left(\mathrm{TL}_{16}=21.1 \mathrm{~mm}\right.$; $\mathrm{TL}_{0}=18.2 \mathrm{~mm} ; \mathrm{p}<0.05$ and $\left.\mathrm{W}_{16}=0.104 \mathrm{~g} ; \mathrm{W}_{0}=0.058 \mathrm{~g} ; \mathrm{p}<0,05\right)$. In both experiments, there was a higher mortality rate in those individuals kept in salinity zero when compared with those ones from salinity 16. Another experiment was set up to assess differences in growth rate between salinity zero and 16. A fifth offspring was split into aquariums with salinities 0 and 16 . During 70 days, individuals were measured to the nearest $\mathrm{mm}$ at regular intervals of five days. Individuals significantly had higher growth rate in salinity 16 than zero. Our laboratorial results seemed to corroborate the field observations showing that the one-sided livebearer Jenynsia multidentata reaches greater total length and weight in brackish, estuarine waters when compared to freshwater.

KEY WORDS. Anablepidae, growth, one-sided livebearer, salinity.

RESUMO. Tendo em vista que as populações de Jenynsia multidentata Jenyns, 1842 aparentemente atingem maiores comprimentos na região estuarina da Lagoa dos Patos quando comparadas com o ambiente límnico adjacente, foi realizado um experimento laboratorial, visando a comparação das taxas de crescimento, do comprimento e peso total dos juvenis da espécie mantidos em duas salinidades (zero e 16). Quatro proles de diferentes fêmeas foram separadas, logo após o parto, em aquários de quatro litros, sendo que metade dos indivíduos de cada prole foi submetida a salinidade zero e a outra metade a salinidade 16. Quatro aquários foram mantidos a temperatura constante de $28^{\circ} \mathrm{C}$ e, outros quatro aquários sofreram modificação da temperatura no décimo oitavo dia de experimento, passando de 28 para $24^{\circ} \mathrm{C}$. Após trinta e oito dias, todos os indivíduos foram medidos e pesados. Os indivíduos mantidos em temperatura constante e salinidade zero, quando comparados com os mantidos em salinidade de 16, apresentaram valores estatisticamente menores de comprimento total $\left(\mathrm{CT}_{16}=23,5 \mathrm{~mm} ; \mathrm{CT}_{0}=\right.$ $20,4 \mathrm{~mm} ; \mathrm{p}<0,05)$ e peso médio $\left(\mathrm{P}_{16}=0,151 \mathrm{~g} ; \mathrm{P}_{0}=0,086 \mathrm{~g} ; \mathrm{p}<0,05\right)$. Os indivíduos mantidos nos aquários que sofreram variação na temperatura também apresentaram diferenças significativas em ambas as medidas $\left(\mathrm{CT}_{16}=21,1\right.$ $\mathrm{mm} ; \mathrm{CT}_{0}=18,2 \mathrm{~mm} ; \mathrm{p}<0,05$ e $\left.\mathrm{P}_{16}=0,104 \mathrm{~g} ; \mathrm{P}_{0}=0,058 \mathrm{~g} ; \mathrm{p}<0,05\right)$. Ao encerrar os experimentos, foi observada maior taxa de mortalidade na salinidade zero quando comparada com a salinidade 16 , em ambos os experimentos. Em um novo experimento, uma quinta prole foi mantida em salinidades de zero e 16 por 70 dias, ocasião em que os indivíduos foram medidos em intervalos regulares de cinco dias. Os indivíduos tiveram maior crescimento em salinidade 16 do que zero. Portanto, em laboratório, o barrigudinho J. multidentata atingi maiores tamanho e peso em água salobra, corroborando as observações obtidas no ambiente natural.

PALAVRAS CHAVE. Anablepidae, barrigudinho, crescimento, salinidade.

Revista Brasileira de Zoologia 22 (3): 780-783, setembro 2005 
Em uma revisão do gênero Jenynsia Gìnther, 1866, GuEdotTi \& WeitZMAN (1996) aplicaram o nome Jenynsia multidentata para a espécie que possui uma ampla distribuição, desde a província do Rio Negro, na Argentina até o Rio de Janeiro, e Jenynsia lineata Jenyns, 1842 para a espécie restrita ao Rio Cebollati, no Uruguai. Contudo, estudos anteriores (CHAO et al. 1985, FontourA et al. 1994, Koblitz \& Andreata 1996, Aranha \& Camaschi 1999) usaram a designação J. lineata para indivíduos que ocorrem fora do Rio Cebollati. Todavia, segundo Guedotti \& Weitzman (1996), tais indivíduos devem ser considerados como J. multidentata.

O barrigudinho, como é conhecido popularmente no Brasil é uma espécie eurihalina, secundária de água doce, ocorrendo em corpos de água doce e estuarinos, e pode ser considerado um dos componentes dominantes da associação de peixes de zonas rasas $(<2 \mathrm{~m})$ do estuário da Lagoa dos Patos, que juntamente com os peixes-rei, juvenis de tainhas e clupeídeos contribuem com mais $90 \%$ do total capturado com rede de arrasto de praia (VIEIRA et al. 1998).

Jenynsia multidentata apresenta fecundação interna, com um período de "gestação" de cinco a seis semanas, e é marcada por dimorfismo sexual. As fêmeas são maiores que os machos e apresentam abertura urogenital, enquanto que os machos possuem a nadadeira anal modificada em gonopódio.

Segundo Fontoura et al. (1994), o barrigudinho atinge tamanhos relativamente maiores em regiões estuarinas quando comparado com populações de ambientes límnicos, sugerindo a hipótese de que águas salobras influenciariam positivamente no crescimento da espécie. Neste sentido, o presente trabalho compara, através de experimentos laboratoriais, a influência da salinidade no crescimento em tamanho e em peso do barrigudinho.

\section{MATERIAL E MÉTODOS}

No dia dezenove de janeiro de 2004, cinco fêmeas grávidas foram coletadas no estuário da Lagoa dos Patos $\left(32^{\circ} 09^{\prime} \mathrm{S}\right.$, $\left.52^{\circ} 06^{\prime} \mathrm{W}\right)$. No momento da coleta a salinidade do local estava em seis. Duas destas fêmeas foram colocadas em água de salinidade zero, e pariram no dia seguinte. Os filhotes de cada uma destas duas fêmeas foram divididos em dois lotes, transportados para aquários de, respectivamente, salinidades zero e 16 , e mantidos à temperatura constante de $28^{\circ} \mathrm{C}$ (Experimento 1). Duas outras fêmeas foram colocadas em água de salinidade 16 , vindo a parir no dia primeiro de fevereiro. Os filhotes de cada uma destas duas fêmeas foram também divididos em dois lotes e transportados para aquários de, respectivamente, salinidades zero e 16 e sofreram, por eventualidade, uma modificação da temperatura no $18^{\circ}$ dia após seu nascimento, quando a temperatura caiu de 28 para $24^{\circ} \mathrm{C}$ e se manteve assim até o final do experimento (Experimento 2).

O alimento, sob a forma de ração balanceada e Artemia sp., foi oferecido ad libtium. O alimento e as fezes restantes nos aquários eram sifonados diariamente, e a água era totalmente renovada a cada cinco dias.
Ao completarem trinta e oito dias de vida, os indivíduos foram secos em papel toalha, medidos seus comprimentos totais (CT) com um paquímetro, em milímetro, e pesados individualmente em balança analítica ao nível de centésimo de grama. Dentro de cada experimento (Experimentos 1 e 2), comparou-se a diferença entre os comprimentos totais e o peso total dos indivíduos mantidos em diferentes salinidades através do teste t-Student.

Com a prole da quinta fêmea, realizou-se o terceiro experimento (Experimento 3), no qual metade da prole foi colocada em salinidade zero e a outra em salinidade 16 . Neste experimento, a cada cinco dias, foram feitas medições do comprimento total de quatro indivíduos durante setenta dias para cada salinidade, os quais foram escolhidos aleatoriamente. Com estes dados foram construídas curvas de crescimento para cada tratamento. Para testar a diferença entre a inclinação das curvas, utilizou-se o método de regressão linear para a comparação de duas retas (ZAR 1984).

\section{RESULTADOS}

A maior tendência de mortalidade ocorreu em salinidade zero (Tab. I). Ao final de trinta e oito dias, no Experimento 1 ocorreu mortalidade de $46,7 \%$ na salinidade zero e $13,3 \%$, na salinidade 16. O Experimento 2 apresentou padrão bastante similar, com mortalidade de $31,6 \%$ e $5,3 \%$ em zero e 16 de salinidade, respectivamente. Portanto, foi observada maior mortalidade na salinidade zero, em ambos os experimentos.

Tabela I. Percentual de mortalidade dos filhotes, após 38 dias, mantidos em salinidade zero e 16 para ambos os experimentos.

\begin{tabular}{crrrr}
\hline Experimento & Sal & $\mathrm{n}$ inicial & $\mathrm{n}$ final & Mortalidade (\%) \\
\hline 1 & 0 & 15 & 8 & 46,7 \\
& 16 & 15 & 13 & 13,3 \\
2 & 0 & 19 & 13 & 31,6 \\
& 16 & 19 & 18 & 5,3 \\
\hline
\end{tabular}

Observa-se que, tanto para o Experimento 1 quanto para o Experimento 2, os indivíduos mantidos em salinidade 16 atingiram, tamanhos significantemente maiores $(\mathrm{p}<0,05)$ quando comparados aos mantidos em salinidade zero (Fig. 1). Os pesos também foram maiores na salinidade 16 do que na salinidade zero ( $\mathrm{p}<0,05$ ), independentemente do experimento (Fig. 2).

Baseado nos dados do Experimento 3 (Fig. 3), observa-se que a taxa de declividade das curvas de crescimento foram diferentes, com o crescimento na salinidade 16 significantemente maior do que o observado na salinidade zero $(\mathrm{p}<0,05)$.

\section{DISCUSSÃO}

O maior crescimento apresentado por $J$. multidentata em salinidade 16 , quando comparada ao crescimento em salinidade 

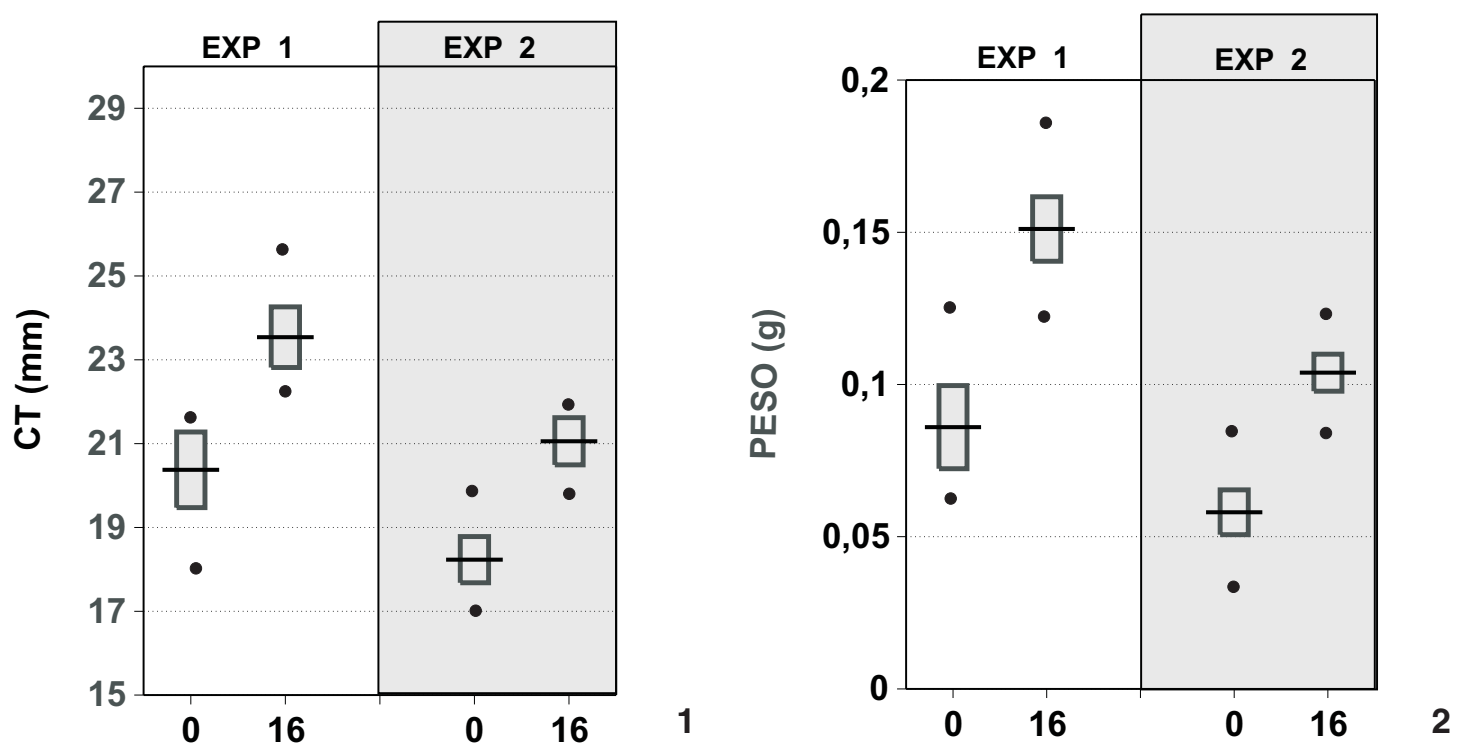

Salinidade

Figuras 1-2. Comparação entre as médias dos: (1) comprimentos totais (CT mm) e (2) pesos (g), atingidos pelos indivíduos de /. multidentata para as salinidades zero e 16 em cada experimento.

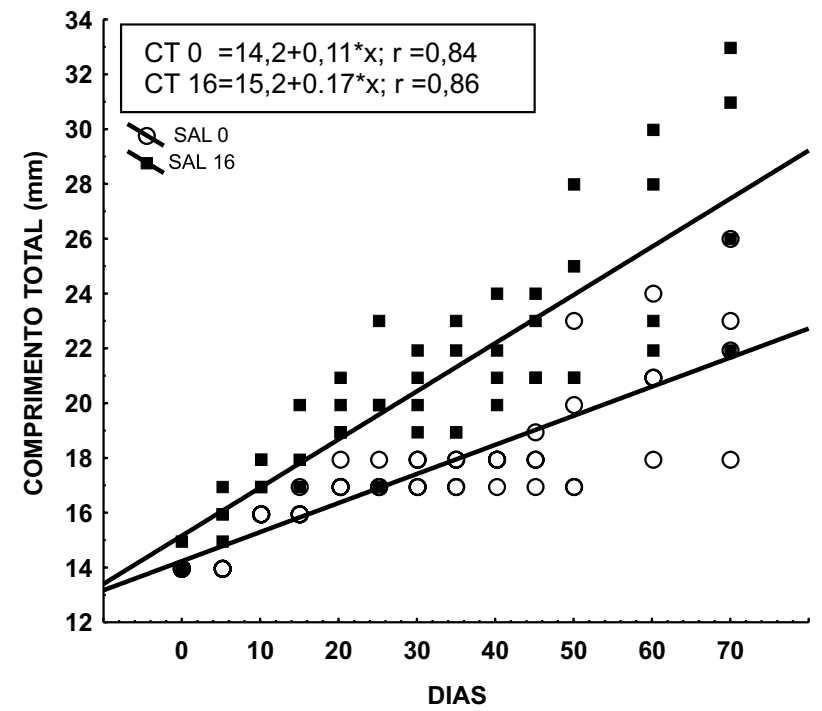

Figura 3. Curvas de crescimento dos filhotes de J. multidentata mantidos durante 70 dias em salinidade zero (circulo aberto) e 16 (quadrado). As observações do comprimento total $(\mathrm{mm})$ de cada indivíduo foram realizadas a cada cinco dias.

zero concordam com as observações de FonToura et al. (1994) que, ao compararem populações de ambientes de água doce e ambientes de água salobra, registram maiores tamanhos para água salobra.
Segundo IMSLAND et al. (2003) freqüentemente, as maiores taxas de crescimento dos peixes foram observadas em condições intermediárias de salinidade (água salobra, 8-20 de salinidade). As análises, geralmente realizadas para testar a influência da temperatura e da salinidade no crescimento de peixes, mostram um maior crescimento nos casos em que se observam as menores atividades da $\mathrm{Na}+\mathrm{K}+$-ATPase. Com isto, os autores sugerem que o crescimento é influenciado pela energia gasta na osmorregulação. Os peixes de água doce são hipotônicos em relação ao meio (MoYle \& CECH 1982) e conseqüentemente é de se esperar que espécies de peixes secundárias de água doce (Myers 1951 apud BerRa 1981), que suportam permanecer em ambientes salobros, tenham maior crescimento nestes ambientes quando comparado com a água doce. As espécies Poecilia reticulata Peters, 1859, Cyprinodon macularius Baird \& Girard, 1853 e Tilapia mossambica (Peters, 1852), assim como J. multidentata, são de origem secundária em água doce, e também apresentam maiores taxas de crescimento em salinidades acima de zero (BRETT 1979). Outra hipótese sugerida na bibliografia é de que a salinidade pode estar aumentando a atividade das células pituitárias produtoras do hormônio de crescimento (GH) (Mancera \& MCCORMick 1998).

Experimentos simples, em que apenas poucos fatores como salinidade e temperatura são controlados, como foi o caso deste trabalho, podem gerar resultados conclusivos. No entanto, outros elementos inerentes aos processos metabólicos das espécies e, que são mais difíceis ou dispendiosos de controlar, podem acrescentar, quando medidos, maior precisão à análise e conclusão dos fatos. Durante nosso experimen- 
to, foi observado que a formação de biofilme, nas paredes dos aquários e junto ao alimento, foi muito mais intensa em água de salinidade zero que nos aquários de salinidade 16 . Com isto observou-se, também, que a espécie deixava de ingerir parte do alimento quando este estava envolto por biofilme, o que ocorria freqüentemente na salinidade zero. Este fato poderia ter contribuído para o menor crescimento da espécie em salinidade zero em nosso experimento, contudo não achamos que o seu efeito tenha afetado no crescimento, já que os aquários eram diariamente limpos e, o alimento era oferecido após essa limpeza, dando tempo para que todos os organismos se alimentassem à vontade antes da formação do biofilme.

Sendo assim, sugerimos que em experimentos futuros sejam utilizados alimentos flutuantes e, que haja um controle da qualidade da água, ao nível de compostos nitrogenados, pois os metabólicos dissolvidos na água podem, além da salinidade, também influenciar no processo de crescimento.

Em conclusão, os juvenis da espécie Jenynsia multidentata apresentam maior crescimento em salinidade 16 quando comparada a salinidade zero, o que vem a corroborar com as observações feitas no ambiente natural, onde os indivíduos capturados em ambientes estuarinos atingem maiores tamanhos quando comparados com a água doce. Estes resultados, associados à maior taxa de sobrevivência observada nos aquários de salinidade 16 , demonstraram que $J$. multidentata esta bem adaptada ao ambiente estuarino, explicando sua elevada abundância no estuário da Lagoa dos Patos (CHAO et al. 1985).

\section{REFERÊNCIAS}

Aranha, J.M.R. \& E.P. CAMASCHI. 1999. Estrutura populacional, aspectos da reprodução e alimentação dos Cyprinodontiformes (Osteichthyes) de um riacho do sudeste do Brasil. Revista Brasileira de Zoologia, Curitiba,16 (1): 637-651.

BERRA, T.M. 1981. An atlas of distribuition of the fresh-water fish families of the word. Lincoln, University of Nebraska, XXI+195p.

Recebido em 16.III.2005; aceito em 22.VIII.2005.
BRETT, J.R. 1979. Environmental factors and growth, p. 599675. In: W.S. Hoar; D.J. Randall \& J.R. Brett (Eds). Fish physiology. London, Academic Press, vol. 8.

Chao, N.L.; L.E. Pereira \& J.P. Vieira. 1985. Estuarine fish community of the Patos Lagoon (Lagoa dos Patos, RS) Brasil. A baseline study, p. 429-450. In: A. YÁñEz-Arancibia (Ed.). Fish community ecology in estuarines and costal lagoons. Towards and ecossistem integration. México, UNAM, 900p.

Fontoura, N.F.; A.S. Braun; D.S. Lewis \& G.D.B. Souto. 1994. Dinâmica populacional da ictiofauna da Lagoa Fortaleza, Cidreira, Rio Grande do Sul II. Jenynsia lineata (Jenyns, 1842) (Teleostei, Anablepidae). Biociências, Porto Alegre, 2: 75-78.

Ghedott, M.J. \& S.H. Weitzman. 1996. A new species of Jenynsia (Cyprinodontiformes: Anablepidae) from Brazil with comments on the composition and taxonomy of the genus. Occasional Papers of the University of Kansas Natural History Museum, Kansas, 179: 1-25.

Imsland, A.K.; S. Gunnarsson; A. Foss \& S.O. Stefansson. 2003. Gill Na+, K+-ATPase activity, plasma chloride and osmolality in juvenile turbot (Scophthalmus maximus) reared at different temperatures and salinities. Aquaculture, Amsterdam, 218: 671-683.

Koblitz, J.L. \& J.V. Andreata. 1996. Análise dos itens alimentares de Jenynsia lineata, Jenyns 1842 (Osteichthyes, Anablepidae) da Lagoa Rodrigo de Freitas, Rio de Janeiro, Brasil. Acta Biologica Leopoldensia, São Leopoldo, 18 (2): 119-127.

Mancera, J.M. \& S.D. MCCormick. 1998. Osmoregulatory actions of the GH/IGH axis in non-salmonid teleosts. Comparative Biochemistry and Physiology, Vancouver, 121: 43-48.

Moyle, P.B. \& J.J. CесH. 1982. Fishes. An introduction to ichthyology. Englewood Cliffs, Prentice-Hall, 597p.

Vieira, J.P.; J.P. Castello \& L.E. Pereira. 1998. Ictiofauna, p. 6068. In: U. Seeliger, C. Odebrecht e J.P. Castello (Eds). Os ecossistemas costeiro e marinho do extremo sul do Brasil. Rio Grande, Ed. Ecoscientia, 341p.

ZAR, J.H. 1984. Biostatistical analysis. New Jersey, $2^{\text {nd }}$ ed., 718p. 